Classification

Physics Abstracts

$7.340-8.822$

\title{
MULTIPLE-PHONON RESONANT RAMAN SCATTERING PROCESSES IN GaSe
}

\section{J. REYDELLET, M. BALKANSKI and J. M. BESSON}

Laboratoire de Physique des Solides,

4, place Jussieu, 75230 Paris Cedex 05, France

(Reçu le 12 mai 1976, accepté le 15 juin 1976)

\begin{abstract}
Résumé. - La résonance Raman de la diffusion multiphonons dans GaSe a été mesurée. Des processus couplant jusqu'à 6 phonons L.O. ont été observés. L'interprétation des résultats doit prendre en compte les processus de couplage anharmonique plutôt que les processus en cascade.

Abstract. - Resonant Raman scattering on L.O. phonon overtones has been measured in GaSe. Coupling of up to six phonons has been observed. Anharmonic coupling processes are shown to be involved and to be dominant over cascade processes.
\end{abstract}

1. Introduction. - Gallium Selenide (GaSe) belongs to the layer chalcogenide III, VI group. Its optical properties make it particularly suitable for the observation of resonant Raman scattering (R.R.S.) on lattice modes through polariton states of the crystal : optical transitions at the gap are allowed for the electric vector of light parallel to the $C$-axis $(E / / C)$ and only weakly allowed by spin-orbit terms for $E \perp C$. In the latter configuration, which is the most accessible experimentally, absorption coefficients are only $\sim 10^{3} \mathrm{~cm}^{-1}$ and the absorption corrections used to compute the Raman cross-section are therefore much less critical than in $\mathrm{CdS}$, for example. At liquid Nitrogen temperatures, the direct exciton whose polariton modes mediate R.R.S., is clearly separated from the continuum. At this temperature, the luminescence originating mainly from the impurity levels, is greatly reduced and broadened in comparison with that observed at liquid Helium temperatures. It can thus be clearly distinguished from Raman scattering from phonons.

For these reasons, resonant Raman scattering from single phonons in GaSe has been observed [1-3] and a quantitative fit of the double resonance peak (in and out - going photon energies) has been performed [1] along existing calculations [4] for polaritonmediated resonance on polar modes (Frölich interaction).

In this letter we report observation of R.R.S. on multiple phonon modes in GaSe. Although many other combination modes may be observed in the resonance region, we will discuss here only the behaviour of the two I.R. active L.O. modes which are at 247 and $256 \mathrm{~cm}^{-1}$ at liquid $\mathrm{N}_{2}$ temperature. The
$247 \mathrm{~A}_{2}^{\prime \prime}$ mode in $\varepsilon$ polytypes does not exhibit combination modes whereas processes can be observed up to six multiple phonons in the case of the $E^{\prime}\left(256 \mathrm{~cm}^{-1}\right.$ mode). The dispersion of the Raman cross section for the two-phonon process has been measured in backscattering geometries both for $E \perp C(Z(X X) Z)$ and $E / / C(X(Z Z) X)$. Multiphonon scattering in the $E / / C$ polarization has been observed independently [5] by another group and interpreted by a cascade process.

Here, we show, on the contrary, that experimental evidence suggests that an anharmonic coupling between the phonons is responsible for multiphonons scattering in GaSe rather than cascade processes. In order to understand the resonance spectra quantitatively, simultaneous emission of phonons at the same point of the diagram must therefore be taken into account and not only, if at all, processes involving successive emission of single modes.

2. Experimental part. - The crystals used were $\varepsilon$-type (either vapour-grown platelets or Bridgeman bulk samples). In the $(X(Z Z) X)$ configuration we used both natural (as-grown) and mechanically polished surfaces parallel to the axis to check for the possibility of surface disorder influence. All samples were grown in the Laboratoire de Luminescence (Université Pierre et Marie Curie).

Experiments were performed at $80 \pm 2 \mathrm{~K}$ in a variable-temperature cryostat and the temperature of the active region was ascertained by measuring the location of the fundamental exciton position [1, 3]. A Spectra-Physics model 370 dyes cell with Rhodamine $6 \mathrm{G}$ was excited by a Coherent Radiation 52 argon 
laser. Spectres were recorded on a Coderg T 800 triple monochromator.

The absorption coefficient in the $E \perp C$ geometry was simultaneously measured under the same conditions and was found to be consistent with previously published results [6]. In the $E / / C$ geometry we used both Irwin' and Hoff's values [2, 7], and values recently measured in our group $\left({ }^{1}\right)$.

Those two sets of data are in reasonable $(20 \%)$ agreement and close enough not do introduce significant uncertainties for our discussion.

3. Results. - Combination frequencies of the $256 \mathrm{~cm}^{-1}$ LO mode ( $E^{\prime}$ infrared active for $E \perp C$ ) have been observed up to 6-LO but no combination bands of the $247 \mathrm{~cm}^{-1}$ mode ( $\mathrm{A}_{2}^{\prime \prime}$ L.O.-infrared active for $E / / C$ ) were present. Figure 1 shows the behaviour of the Raman cross-section for incident and scattered light perpendicular to the $C$ axis $(Z(X X) Z)$ at $80 \mathrm{~K}$. In this geometry, although the Raman efficiency is lower than for light polarized parallel to the $C$ axis, the precision of data is higher since the absorption coefficient is directly accessible and can be taken into account without difficulty. This point is important for our discussion on the possibility of supplementary peaks in the Raman cross section (\$4). Figure 2 shows the behaviour of the Raman tensor for incident and scattered light parallel to the $C$ axis $(X(Z Z) X)$ for 2,3 and 4 phonon processes. It should be noted here that the absolute magnitudes of the Raman tensor in the two geometries should be compared only quali-

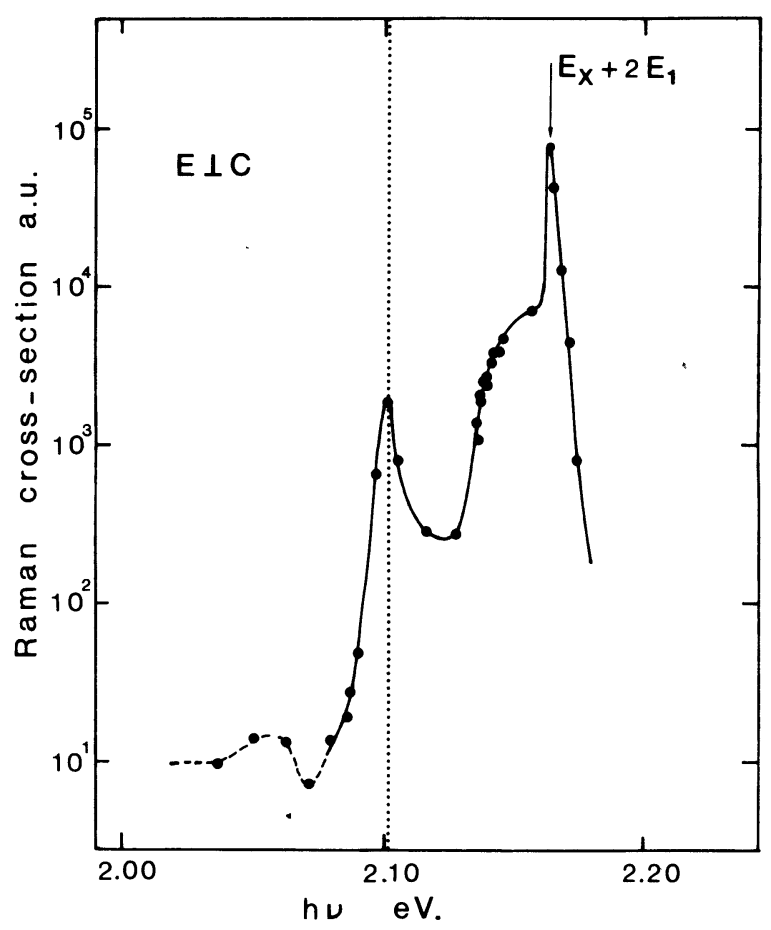

FIG. 1. - Raman cross-section for 2-phonon processes in the $Z(X X) Z$ geometry $(E \perp C)$. Same conditions as figure 2 .

( $\left.{ }^{1}\right)$ Le Toullec, R. et al., Private communication.

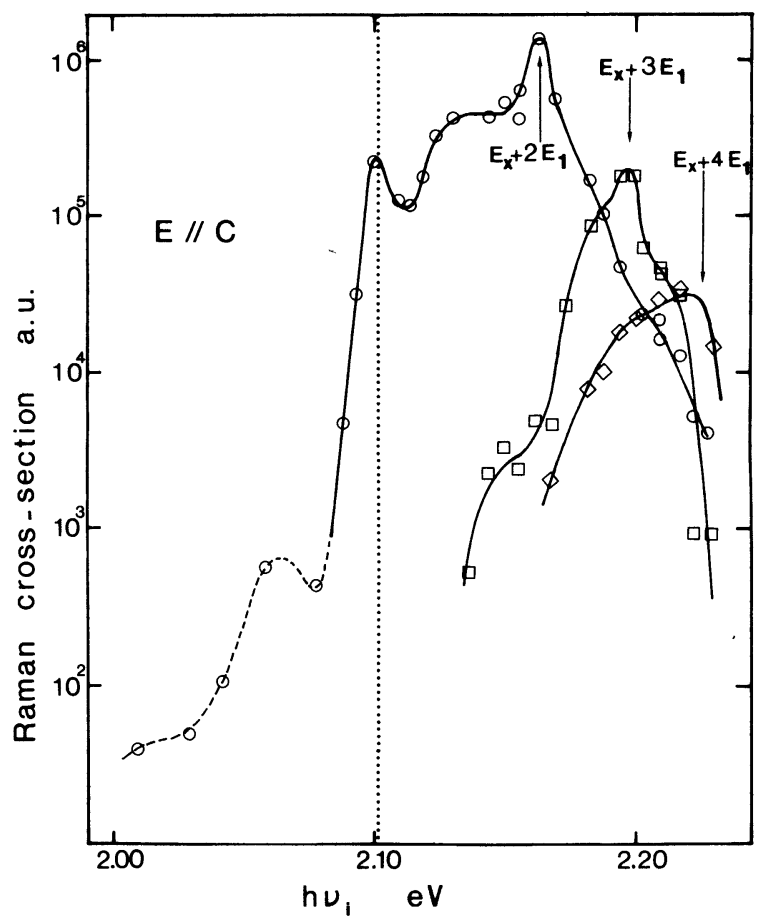

FIG. 2. - Raman cross-section of multiphonon processes in the $X(Z Z) X$ geometry $(E / / C) . T=80 \mathrm{~K} . \bigcirc: 2$-phonon processes for the $E^{\prime}$ (L.O. C $-256 \mathrm{~cm}^{-1}$ ) phonon. $\square:$ 3-phonon processes. $\diamond:$ 4-phonon processes. $E_{X}$ : exciton energy (dotted line) at $80 \mathrm{~K}$ : $2.102 \mathrm{eV} . E_{1}$ : energy of the $\mathrm{e}^{\prime}$ phonon.

tatively, although, the Raman cross-section is indeed, smaller for $E \perp C$ (Fig. 1) than for $E / / C$ (Fig. 2).

The $E \perp C$ resonance is clearly sharper than the $E / / C$ resonance and this can be explained on the basis of the optical transition processes in the crystal for both polarizations. We will now discuss the following experimental results :

1) Up to six (and possibly more) overtones of the $256 \mathrm{~cm}^{-1}$ LO phonon can be observed while no combination from the $247 \mathrm{~cm}^{-1}$ mode is present.

2) This sharply contrasts with the one-phonon resonance process where these two modes have comparable behaviour [1-3].

3) The intensity of $n$-phonon processes decreases very sharply with the order $n$.

In this comparison, we take a representative parameter to measure the intensity of a given process to be the total intensity of the Raman cross-section integrated over energy $I=\int_{E} S \mathrm{~d} E$. On the contrary, the intensity of a peak of Raman cross-section depends largely upon the relevant damping constant, and values of the Raman cross-section taken at an arbitrary wavelength are, in general, meaningless. In our case, for example, it is found that at $h v=2.210 \mathrm{eV}$, the relative magnitudes of the $n=2,3$ and 4-LO phonon processes are respectively 1,2 and 5 . If this were taken as a measure of the absolute intensity of the process, it would lead to wholly unjustified conclusions. 
If we take $\int_{E} S \mathrm{~d} E$ to be unity for $n=2$, we have the following values (Table).

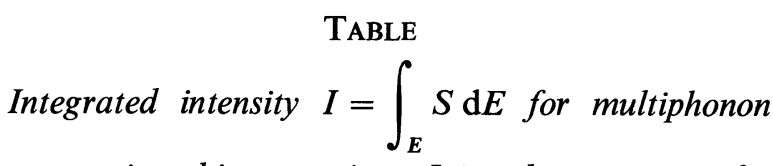
processes in arbitrary units : $I$ is taken as unity for the $2 \times E_{1}\left(2 \times 256 \mathrm{~cm}^{-1}\right)$ process.

Order of process $n \quad 2 \quad 3$

Integrated intensity

$I=\int_{E} S \mathrm{~d} E$

$16.5 \times 10^{-2} 5.8 \times 10^{-3}<8 \times 10^{-4}$

The rather rapid decrease of $I$ with $n$ will be used to argument about the proposed scattering mechanism.

4) Infrared measurements [8] on GaSe show a strong two-phonon absorption band at $2 \times 256 \mathrm{~cm}^{-1}$ and none in the region of $2 \times 247 \mathrm{~cm}^{-1}$.

Since multiphonon infrared absorption is mediated by anharmonic terms in the crystal potential, this indicates that the displacement pattern of the $\mathrm{E}^{\prime}$ mode $\left(256 \mathrm{~cm}^{-1}\right)$ involves much stronger anharmonic forces than the $A_{2}^{\prime \prime}\left(247 \mathrm{~cm}^{-1}\right)$.

This conclusion being drawn from infrared absorption is strictly valid for phonons at the edge of the Brillouin zone whence the main part of the process originates (high density of states). But it is qualitatively correct also for phonons at the center of the zone (Raman scattering) since the direction of vibration is the same : parallel to the $C$-axis for the $\mathrm{A}_{2}^{\prime \prime}$ mode, perpendicular for the $E^{\prime}$ mode. Finally, the fact that the combination frequency for edge-of-thezone phonons (infrared absorption [8] is close to that for center-of-the zone modes follows from the flatness of dispersions curves for this branch [9]).

4. Discussion. - The main features of the dispersion of the Raman cross-section are analogous for multiple phonons to those we $[1,3]$ have already published for single phonon processes. Strong maxima are observed at the ingoing photon energy equal to exciton plus $n$-phonons energy (outgoing resonance). Although a shoulder on the low-frequency side of the outgoing resonance peak appears consistently in one-and-multiple-phonon spectra above $2140 \mathrm{meV}$, we will not try to interprete it quantitatively here. We have shown that for one-phonon spectra [1] it could be explained by taking into account $n=2$ and $n=3$ exciton levels as an intermediate state in addition to the fundamental $n=1$ exciton level; the contribution of the band gap continuum, although much weaker, may also be considered. The shoulder in multiple phonon resonance curves is much more pronounced and only a quantitative fit will permit one to determine whether its location close to the direct band gap is fortuitous or not. Nevertheless, the precision of our measurements is sufficient in the
$E \perp C$ configuration (Fig. 2) to exclude the possibility of its assignment to the $E_{X}+E_{1}$ pole which would be present in a cascade process. Several processes which may be involved in multiple phonon resonance lead to different diagrams describing them. In the multiple LO phonon scattering in CdS, a cascade theory was used [10]. This approach involves successive emission of single phonon through 3-particles processes and neglects processes in which more than one LO phonon is emitted simultaneously. This theory does not seem to apply here, for the following reasons :

1) It does not explain why the $A_{2}^{\prime \prime}\left(247 \mathrm{~cm}^{-1}\right)$ gives no multiple combinations while the one-phonon mode has a strong resonance. The fact that this $A_{2}^{\prime \prime}$ mode is Raman-forbidden should not prevent it from exhibiting cascade processes (cf. $\mathrm{CdS}$, for example).

2) The decrease in magnitude for the successive multiple combination resonances given at point 3 ), paragraph 3 , is very different from those expected from this process.

3) This theory predicts intermediate maxima between the in-and-outgoing resonances. No evidence for such maxima has been seen in our experiments particularly for $E \perp C$ (Fig. 2) where the energy expected for an intermediate maximum at

$$
E_{X}+E_{1}=2.132 \mathrm{eV}
$$

is actually close to a minimum in $\mathbf{S}$.

In contrast with this proposal (cascade processes), the following features do point to simultaneous multiple phonon emission :

1) Multiple phonon processes which are observed in infrared absorption [8] are related to anharmonic coupling through higher-order terms in the expression for the total crystal potential. Those measurements do show that two-phonon processes are strong at $2 \times 256 \mathrm{~cm}^{-1}\left(512 \mathrm{~cm}^{-1}\right)$ and not observed at $2 \times 247 \mathrm{~cm}^{-1}$. This anharmonic coupling process contributing to the Raman scattering is expected to be much stronger for $2 \times 256 \mathrm{~cm}^{-1}$ than $2 \times 247 \mathrm{~cm}^{-1}$, as observed in our experiments.

2) The rapid decrease in intensity for the first combination of the $256 \mathrm{~cm}^{-1}$ mode is completely expected for simultaneous emission of several phonons since the order of magnitude of the successive terms in the expression of the crystal potential is expected to decrease with their order. This is not the case for a cascade process [10].

Finally, the sharper resonance shape for $E \perp C$ (Fig. 2) than for $E / / C$ (Fig. 1) is to be related to the nature of the optical transition at the gap. It is almost forbidden for $E \perp C$ and rendered only weakly allowed by the spin-orbit interaction. Since, the principal intermediate process (electron-hole pair or free exciton creation) which becomes a real process at resonance is almost forbidden, we expect the lifetime to be long and the damping constant small for 
the process. This had previously been observed for dipole-forbidden exciton states in $\mathrm{Cu}_{2} \mathrm{O}$ [11], exhibiting a remarkably sharp resonance. In $\mathrm{GaSe}$, the transition process is such that it can be observed in resonance for an allowed and a quasi-forbidden polarization.

5. Conclusion. - All the experimental evidence presented here points to multiple phonon processes involving, at least in part, anharmonic terms in the crystal potential and shows that this process must be taken into account for a numerical fit with the experiments. It is also an indication of the aniso- tropy of anharmonic forces in layer crystals which is expected to result from the difference in nature and magnitude of inter-and intra-layer forces, but has not been previously reported. A quantitative fit with experiment which takes into account anharmonic coupling is under way and will be reported in a later paper.

Aknowledgments. - We thank J. Camassel (CEESUniversité des Sciences et Techniques du Languedoc) and his co-workers for a preprint of their paper on the subject and for stimulating discussions.

\section{References}

[1] Reydellet, J., Besson, J. M., Solid State Commun. 17 (1975) 23.

[2] Hoff, R. M., Irwin, J. C., Phys. Rev. B 10 (1974) 3464.

[3] Reydellet, J., Besson, J. M., Proceedings of the International Conference on Light Scattering (Campinas 1975) p. 59 (Flammarion, Paris) 1976.

[4] Ganguly, A. K., Birman, J. L., Phys. Rev. 162 (1967) 806.

[5] Camassel, J., Chiang, T. C., Shen, Y. R., Vortchkovsky, J. P., AMER, N. M., to be published in Solid State Commun. (1976).

[6] Khelladi, F. Z., Thesis, Paris VI University (1971) Unpublished and :
Bourdon, A., Khelladi, F. Z., Solid State Commun. 9 (1971) 1715.

[7] Hoff, R. M., Ph. D : Thesis, Simon Fraser University (1975) Unpublished.

[8] Kuroda, N., Nishina, Y., Fukurol, T., J. Phys. Soc. Japan 24 (1968) 214.

[9] Jandl, S., Brebner, J. L., Powell, B. M., Phys. Rev. B 13 (1976) 686.

[10] Martin, R. M., Varma, C. M., Phys. Rev. Lett. 26 (1971) 1241.

[11] Compana, A., Cummins, H. Z., Phys. Rev. Lett. 31 (1973) 41. 